\title{
Effect of Using Smartphone during Breast-feeding
}

\author{
Haruka Nakagawa \\ Kobe University \\ Kobe, Japan \\ Tsutomu Terada \\ Kobe University \\ Kobe, Japan
}

\author{
Sachine Yoshida \\ Toho University \\ Tokyo, Japan \\ Hiromasa Funato \\ Toho University \\ Tokyo, Japan
}

\author{
Ayumi Ohnishi \\ Kobe University \\ Kobe, Japan \\ Masahiko Tsukamoto
Kobe University
Kobe, Japan
}

\begin{abstract}
With the spread of smartphones, mothers who operate smartphones while breast-feeding are increasing. This kind of activities are sometimes defined as a bad behavior although this is one of few repose in parenting. In this paper, we investigate if the use of smartphone affects the breast-feeding from the viewpoints of the mother's posture and the quality of communication between the mother and the baby. We measure the behavior of the mother with/without smartphone using wearable sensors and video camera. As a result of the survey, sensor data did not show the significant difference in the mother's posture. In the observation of the video camera, the inclination of a mother's back was different depending on the presence or absence of a smartphone operation. As a result of research on communication with infants, it was longer for mothers to notice changes in their baby while operating smartphones. In the future, in order to reduce mother's stress, we will consider how to operate smartphones properly, instead of prohibiting operation of smartphones for nursing care.
\end{abstract}

\section{KEYWORDS}

infant, smartphone, breast-feeding, wellbeing

\section{ACM Reference Format:}

Haruka Nakagawa, Sachine Yoshida, Ayumi Ohnishi, Tsutomu Terada, Hiromasa Funato, and Masahiko Tsukamoto. 2019. Effect of Using Smartphone during Breast-feeding. In Adjunct Proceedings of the 2019 ACM International foint Conference on Pervasive and Ubiquitous Computing and the 2019 International Symposium on Wearable Computers (UbiComp/ISWC '19 Adjunct), September 9-13, 2019, London, United Kingdom. ACM, New York, NY, USA, 5 pages. https://doi.org/10.1145/3341162.3344840

\section{INTRODUCTION}

Smartphones are now in widespread use across generations and support our daily lives. Mothers with infants have little time to rest in parenting, and sometimes use breast-feeding time to use their smartphones. On the other hand, in the concept of traditional parenting, it is said that smartphone operation during breast-feeding

Permission to make digital or hard copies of all or part of this work for personal or classroom use is granted without fee provided that copies are not made or distributed for profit or commercial advantage and that copies bear this notice and the full citation on the first page. Copyrights for components of this work owned by others than ACM must be honored. Abstracting with credit is permitted. To copy otherwise, or republish, to post on servers or to redistribute to lists, requires prior specific permission and/or a fee. Request permissions from permissions@acm.org.

UbiComp/ISWC'19 Adjunct, September 9-13, 2019, London, United Kingdom

() 2019 Association for Computing Machinery.

ACM ISBN 978-1-4503-6869-8/19/09.. \$15.00

https://doi.org/10.1145/3341162.3344840 is not good for infants. However, no clear reason has been investigated about that. New technologies like smartphones make our lives convenient. From the viewpoint of well-being, it is important not to reject advanced technology without a clear reason. Considering mothers' stress, it is desirable to support mothers to be able to use their smartphone as much as possible without adversely affecting children. A conventional research suggests mother's smartphone operation during eating worsen parent-child relationships in some cases[1]. However, as far as we know, there is no research focusing on smartphone operation during breast-feeding.

By using a smartphone while breast-feeding, there is a possibility that a mother's posture and communication with their baby become impaired, which adversely affect parents and children. The effects are, for example, parent-child relationships, physical pain like backache in the mother, infant's satisfaction with breast-feeding, poor sleep quality. The reason for this effect is probably because there is something different between the presence and absence of smartphone operation. The differences are, for example, a mother's breast-feeding posture and mother-baby communication. In this study, we investigate whether there is a difference in a mother's posture and communication with their baby depending on the presence or absence of smartphone operation during lactation.

\section{RELATED RESEARCH}

there are studies on a relationship between breastfeeding and the healthy growth of infants. Endang at al. suggests that breastfeeding is a protective factor against stunts[2]. Because breastfeeding is important, there are also studies that promote it. Guqin et al. Showed that information support and behavioral interventions can effectively improve anxiety and depression, knowledge of breastfeeding, breastfeeding rates in primiparous women[3].

Also, there are researches on the effect of smartphone abuse on the parent-child relationship. Jenny et al. suggests that smartphone operation during eating may worsen mother-child relationships[1]. Brandon et al. investigates whether the use of inappropriate technology by mothers and fathers is related to parent-child interaction, and shows that technical interventions are related to children's problem atic behavior[4]. Also, Sarah et al. suggested that the use of mobile devices by parent would affect the quality of parent-child interaction, and asserted the need for continuous research on the role of technology in infant development[5]. These 


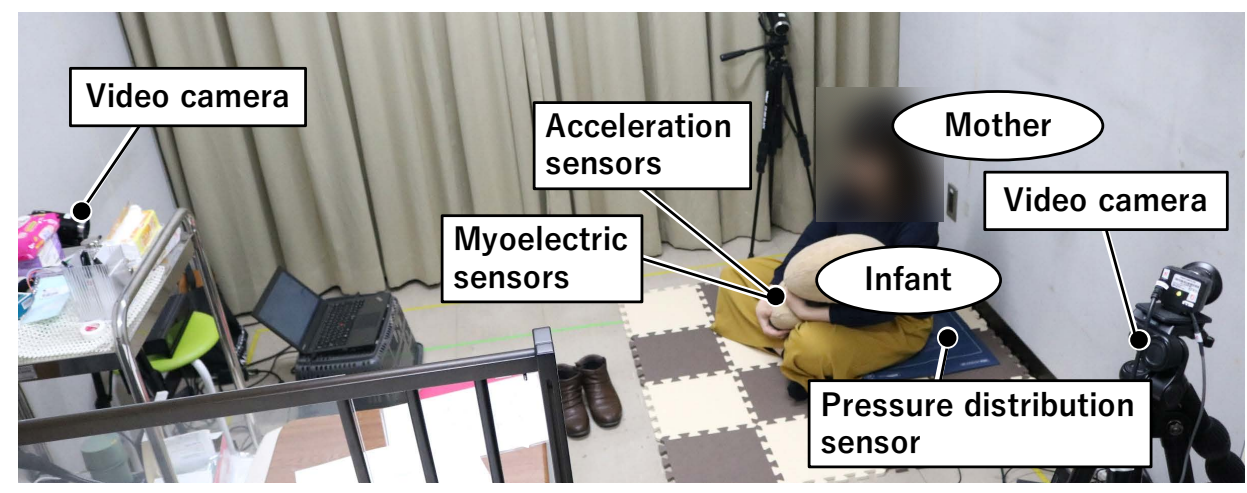

Figure 1: The system configuration

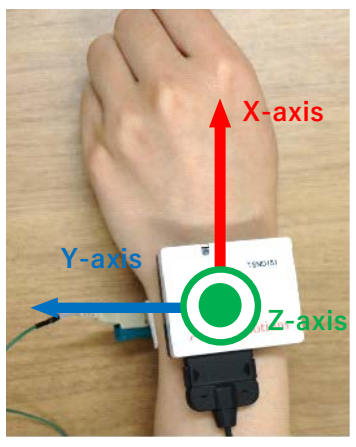

Acceleration and angular velocity sensor

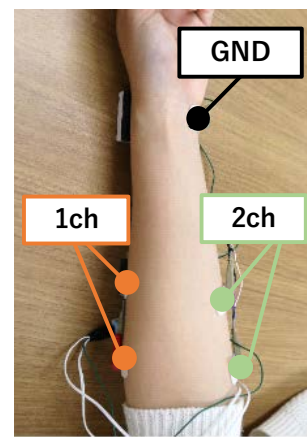

Myoelectric sensor
Figure 2: Mounting diagram

studies suggest that the use of mobile devices can affect the richness of parent-child relationships. However, no definitive conclusions have been reached and no studies have focused on smartphone operation during breast-feeding as far as we know. Therefore this paper focuses on smartphone operation at the time of breast-feeding.

\section{METHOD}

\subsection{Data acquisition}

Figure 1 shows the configuration of the system used for verification of the mother's posture during breast-feeding and communication with the baby. Changes in mother's posture are measured using several sensors and a video camera. Arm movements are attached to the mother's wrists with acceleration and angular velocity sensors to acquire 3-axis acceleration and angular velocity data (Figure 2). The degree of force applied by both arms of the mother is obtained by attaching a two-channel myoelectric sensor to both arms of the mother and acquiring data (Figure 2). A change in the position of the center of gravity of the mother is carried out by installing a seat sensor on the seating surface and acquiring and analyzing the pressure distribution(Figure 3). During the experiment, two video cameras are installed on the front and the side

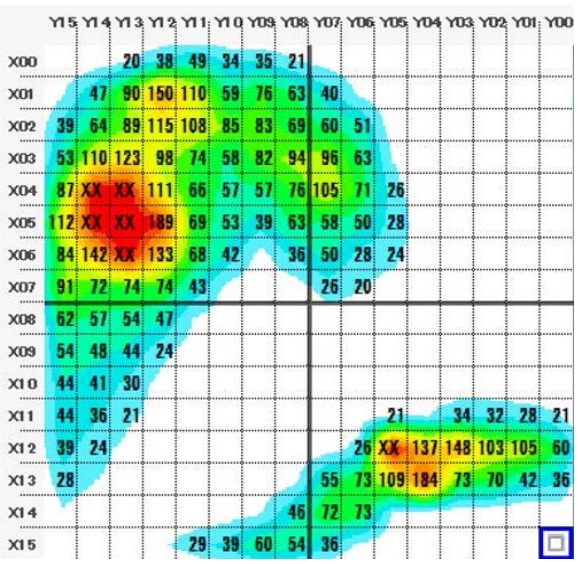

Figure 3: Pressure distribution acquired from sheet sensor

of the subject and observed. Communication with the baby is observed from the two video cameras installed. In order to know the mother's thoughts, subjects were asked a questionnaire survey on smartphone operation during usual breast-feeding. The questions are the frequency of use, the time of use, the baby's reaction, the presence of guilt, the content of the operation, and the attention when operating the smartphone. In the experiment, the subjects took three types of breast-feeding postures: breast-feeding only, breast-feeding while reading news on a smartphone, and breastfeeding while typing characters on a smartphone. The posture was measured for 1 minute and 30 seconds each. The mother's posture was uniform as Figure 1. The subjects are seven pairs of babies and their mothers. At the time of the experiment, the infant was 3 to 6 months old. This experiment was conducted with the approval of the Ethics Committee.

\subsection{Evaluation method}

The following three points were evaluated. The first is the posture of the mother. Each sensor value is graphed and evaluated whether there is a big difference in the waveform depending on the presence or absence of the smartphone operation. In addition, the results of observation with a video camera are also summarized. 
Table 1: The result of the angle of mother's back bends

\begin{tabular}{l|ccccccc}
\hline \hline & \multicolumn{1}{|c}{ Subject } \\
& $\mathrm{A}$ & $\mathrm{B}$ & $\mathrm{C}$ & $\mathrm{D}$ & $\mathrm{E}$ & $\mathrm{F}$ & $\mathrm{G}$ \\
\hline With smartphone operation $\theta\left[{ }^{\circ}\right]$ & 59 & 59 & 65 & 50 & 82 & 60 & 78 \\
No smartphone operation $\theta\left[{ }^{\circ}\right]$ & 62 & 62 & 59 & 53 & 80 & 63 & 80 \\
\hline
\end{tabular}

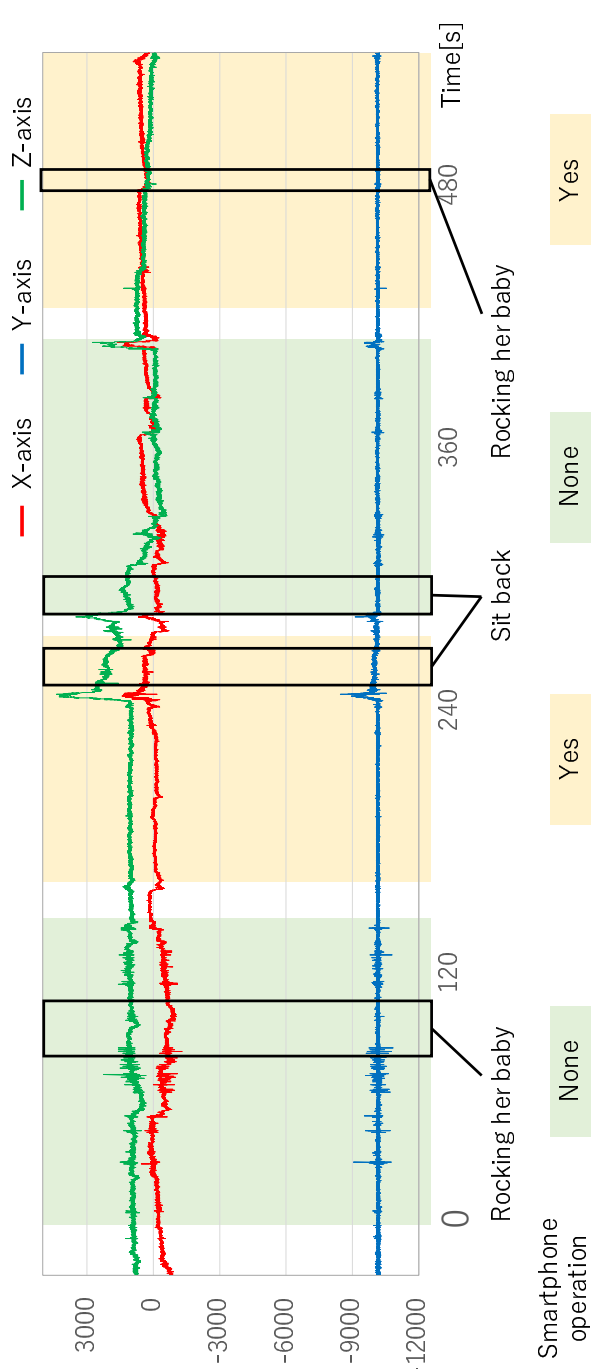

Figure 4: The result of an acceleration sensor for the left wrist of the subject $D$

Measure and evaluate the time it takes for the mother to notice the change in the baby. This time, it is considered as change in the baby that the infant stops drinking milk. We measured the time from the moment the baby left the mother's chest to the moment the mother's forehead turned to the baby. The third is the result of the questionnaire survey. By knowing the situation during normal lactation, it is possible to know if there is a difference on the experimental day. In addition, a mother's feelings can be known. The

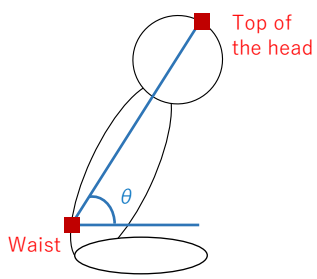

Figure 5: Angle of the mother's back bends

question items are frequency of use, time of use, presence of guilt, infant's reaction, content of operation, and attention in operation.

\section{RESULTS}

\subsection{The posture of the mother}

Detailed analysis on the sensor is omitted because it has no space. The entire waveform of each acquired mother sensor value is observed, and it is evaluated whether the waveform differs greatly depending on the presence or absence of the smartphone operation. As a result, there was no significant difference common to all subjects by the presence or absence of smartphone operation. Next, a waveform of an acceleration sensor for the left wrist of the subject D is shown in Figure 4. Subject D held an infant with his left arm from the beginning to the end of the experiment. As shown in Figure 4, there were times when there were major changes in the waveform. When the video at that time was confirmed, some actions of the mother were confirmed. It was an act of the mother reseating and the mother rocking her baby. These actions were confirmed regardless of the smartphone operation. Similarly, there were no obvious differences in other sensor data and the subject's waveform regardless of the smartphone operation.

Next, the state of the experiment was observed from the video. From the video, it was assumed that the inclination of the mother's back might be different depending on the presence or absence of the smartphone operation. Therefore, the video camera on the side of subjects was evaluated. Thirty seconds after the task started, the angle $\theta$ between the floor and straight line connecting the mother's waist and the top of the head are cutted out and measured.(Figure 5).

Thirty seconds after the task started, the subject was extracted as a calm and not tired time. There was no continuous change in back tilt during the task. This was also judged from the data of the seat sensor. The results are shown in the Figure 1. Among the seven subjects, five subjects had a larger angle with the floor when they had smartphone operation. And when They were not using the smartphone, they bent their back and took a posture to look into the baby's face. On the other hand, subjects $\mathrm{C}$ and $\mathrm{E}$ had their backs extended when the smartphone was not operated, and their 
Table 2: Time for a mother to notice that her baby stop breastfeeding

\begin{tabular}{c|ccc}
\hline \hline & \multicolumn{3}{|c}{ Subject } \\
& C & E & G \\
\hline With smartphone operation [s] & 1.74 & 5.96 & 0.79 \\
No smartphone operation [s] & 0 & 0 & 0 \\
\hline
\end{tabular}

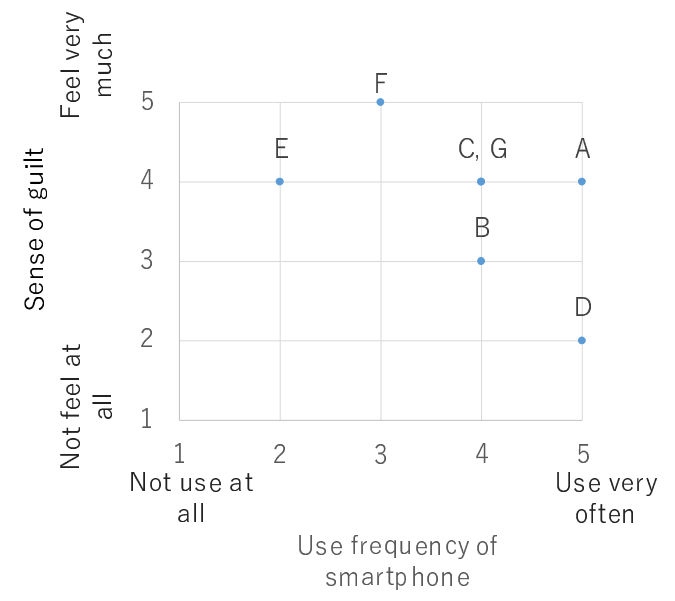

Figure 6: Result of questionnaire survey

angle with the floor became bigger. The reason is that the subject $\mathrm{C}$ did not hold the smartphone by hand, was placed on her foot and operated.

\subsection{Time for mother to notice that baby stop breastfeeding}

The time until the mother notices that the baby has stopped drinking milk is shown in the Figure 2. However, subjects A, B, D, and $F$ were excluded because the infant never left the mother's chest. The zero second result indicates that the mother was always facing before the baby stopped drinking. Most of the subjects looked at the baby's face while not operating the smartphone. As a result, the time it took for the mother to notice all three of them resulted in 0 seconds. This result indicates that it takes more time for the mother to notice that the baby has stopped drinking when he is using the smartphone than when he is not using the smartphone.

\subsection{Result of questionnaire survey}

The results of the questionnaire survey are shown in Figure 6. There were no mothers who did not feel guilty at all during breastfeeding smartphone operation. However, in response to the question whether or not to operate the smartphone during lactation, all subjects answered that they sometimes operate a smartphone. This result indicates that the mother wants to operate the smartphone while breast-feeding. Therefore, it is possible that mothers may feel stress if they prohibit smartphone operation during nursing. Although they felt guilty, all subjects answered that they operated the smartphone during lactation and four out of seven mothers feel that changes in their baby appear as a result of smartphone operation. The remaining three found that they did not feel that the change in the baby appeared by operating the smartphone.

\section{DISCUSSION}

From the results of the experiment, it was found that the mother's posture and the communication with the infant differ depending on the presence or absence of the smartphone operation during breast-feeding. It was suggested that in order to look at the baby well if there is no smartphone operation, the back is rounded and that the change of the baby is noticed quickly. There is a possibility that parent-child communication may be disturbed by the long time to notice the change of the infant. However, changes in the mother's back angle are small, and further investigation is needed to conclude that there is a significant difference between the presence and absence of smartphone operation. In addition, it was found from the result of the questionnaire survey that there was no mother who did not feel guilty at all during nursing smartphone operation, but there was also no mother who did not operate smartphone at all. Several future system configurations were discussed. This time, in the evaluation of the mother's posture, the inclination of the back was analyzed from the video camera image. In the future, it will be considered using sensors. Also, for communication with the infant, there was a mother who did not see the baby even though she was not operating the smartphone in the preliminary experiment. However, mothers of this subject was basically looking at their baby when not using the smartphone. Based on the presence or absence of smartphone operation, we examined differences common to all subjects. In the future, each subject will be evaluated in detail.

\section{CONCLUSION}

This paper investigated whether mother's posture and communication with infant were different depending on the presence or absence of smartphone operation during breast-feeding. It turned out that the time when the mother notices the change of the baby is related to the presence or absence of smartphone operation during lactation. From this result, it is possible that the change caused by smartphone operation during breast-feeding may affect the mother and infant.

\section{REFERENCES}

[1] Jenny Radesky, Christy Leung, Danielle Appugliese, Alison L. Miller, Julie C. Lumeng, Katherine L. Rosenblum: Maternal Mental Representations of the Child and Mobile Phone Use During Parent-Child Mealtimes. Fournal of Developmental \& Behavioral Pediatrics, Vol. 39, No. 4, pp. 310-317 (2018).

[2] Endang Dewi Lestari, Faraissa Hasanah, Novianto Adi Nugroho: Correlation between non-exclusive breastfeeding and low birth weight to stunting in children. Paediatrica Indonesiana, Vol. 58, No. 3, pp. 123-127 (2018).

[3] Guqin Li, Jianxiang Cong, Li Li, Yongmei Li: Effects of nursing with information support and behavior intervention on lactation and breastfeeding success rate for primiparas. International fournal of Clinical and Experimental Medicine, Vol. 11, No. 3, pp. 2617-2623 (2018).

[4] Brandon T. McDaniel, Jenny S. Radesky: Technoference: Parent Distraction With Technology and Associations With Child Behavior Problems. Child Development, Vol. 89, No. 1, pp. 100-109 (2018).

[5] Sarah Myruski, O. A. Gulyayeva, Samantha L Birk, Koraly Prez-Edgar, Kristin A. Buss, Tracy A Dennis-Tiwary: Digital disruption? Maternal mobile device use is related to infant social- emotional functioning. Developmental Science, Vol. 21, No. 4, e12610 (2018). 